\title{
Gas Chromatographic Analysis of Total Fatty Acids in Cider
}

\author{
Domingo Blanco-Gomis, ${ }^{* \dagger}{ }^{\dagger}$ J uan J . Mangas Alonso, ${ }^{\ddagger}$ Inmaculada Margolles Cabrales, ${ }^{\dagger}$ and \\ Pilar Arias Abrodo ${ }^{\dagger}$
}

\begin{abstract}
Departamento de Química Física y Analítica, Universidad de Oviedo, E-33006 Oviedo, Spain, and Servicio Regional de Investigación y Desarrollo Agroalimentario, Apartado 13, E-333000 Villaviciosa, Spain
\end{abstract}

\begin{abstract}
This paper reports the composition of total fatty acids in an apple beverage, cider. Fatty acids are present in the free or esterified form and contribute to both the flavor and foam properties of cider. Fatty acids were separated and identified as methyl esters by GC-MS, and 12 of these were subsequently determined by GC-FID. The major fatty acids found in cider were caproic, caprylic, capric, and palmitic acid, the saturated acids predominating over the unsaturated ones. The proposed method was applied to 59 ciders from three consecutive harvests (1996, 1997, and 1998), which were made by 19 cider-makers from the region of Asturias (Spain). Linear discriminant analysis of fatty acids in these samples allowed selection of palmitoleic, pentadecanoic, linoleic, myristic, and linolenic acid as the most predictive variables to differentiate ciders made from apples grown in the Asturias region (1997 harvest) and ciders made from apples grown outside this region (1996 and 1998 harvests).
\end{abstract}

Keywords: Cider; fatty acids; harvest; multivariate techniques

\section{INTRODUCTION}

Nowadays, there exists a great deal of widespread interest among consumers concerning the nutritious and sensory qualities of food. Lipids are partly responsible for the physical and chemical properties of food. The major lipids of interest in nourishment are fatty acid esters, and many lipid properties in food are explained in terms of their fatty acid composition.

Among alcoholic beverages, lipids and, more specifically, fatty acids have an important influence on the fermentation process as well as on the sensory properties of the final products. Therefore, control of the fatty acid content would contribute to obtaining alcoholic beverages of improved quality.

The fatty acids found in cider come directly from apples, but they can also be formed during alcoholic fermentation. They are present in two forms: free and bound. Both contribute to flavor: the volatile fatty acids directly (1) and the unsaturated ones in an indirect way, as precursors of volatile compounds such as al dehydes and alcohols (2). At the same time, medium-chain fatty acids play a key role in causing arrested fermentations because they are toxic to yeast and mal olactic bacteria and can inhibit the fermentation process $(3,4)$.

On the other hand, studies carried out in beverages such as sparkling wine and beer have revealed that fatty acids contribute to foam formation and stability $(3,5-8)$. At present, there are no similar studies with regard to cider; for this reason in this paper we propose a method for determining the fatty acid composition in cider.

The cider apple tree gives a biennial crop. In odd years, practically all of the apples used in the Asturian cider industry are grown in the region; in contrast, cider manufactured in even years is made from apples

\footnotetext{
† Universidad de Oviedo.

‡ Servicio Regional de Investigación y Desarrollo Agroalimentario.
}

harvested in other regions of Spain, France, and countries of Central Europe. Ther efore, to establish a quality denomination of origin for Asturian cider, it is very important to optimize analytical strategies that allow characterization of the cider made from apples harvested in the Asturian region. In this study, 59 ciders from three consecutive harvests (1996, 1997, and 1998) were analyzed to evaluate fatty acid profiles and to ascertain if these analytes allowed ciders made from apples harvested in the region of Asturias (1997 harvest) to be differentiated. Fatty acids have also been used to characterize food. For instance, the analysis of minor and major fatty acids in almonds allowed Spanish almond cultivars to be characterized $(9,10)$.

The analytical method proposed for the analysis of fatty acids includes extraction, derivatization to methyl esters, and gas chromatography (GC). The derivatization reaction to obtain fatty acid methyl esters (FAMEs) includes the selection of a derivatization reagent, the study of the period of contact between the fatty acids and this reagent, and the temperature of the reaction. We used methanolic $\mathrm{BF}_{3}$ in combination with sodium methoxide to esterify free and bound fatty acids.

\section{MATERIALS AND METHODS}

Chemicals. Individual fatty acids of a purity of $>98 \%$ were purchased from Sigma (St. Louis, MO). Solvents, hexane for organic trace analysis and chloroform, were obtained from Merck (Darmstadt, Germany) and methanol from Romil (Cambridge, U.K.). BF 3 in methanol was supplied by Sigma and sodium methoxide from Aldrich (Steinheim, Germany).

A stock solution of fatty acids was prepared by dissolving $\sim 100 \mathrm{mg}$ of each fatty acid in $10 \mathrm{~mL}$ of methanol.

Sample Preparation and Derivatization. Fifty-nine typical ciders supplied by traditional cider-makers from the region of Asturias from three consecutive harvests (1996, 1997, and 1998) were employed in this study.

A volume of each internal standard (pelargonic and margaric acid) was added to $50 \mathrm{~mL}$ of the sample. 
Total lipids from samples were extracted at room temperature with a mixture of chlorofom and methanol $(2: 1, \mathrm{v} / \mathrm{v})$. The organic phase was separated and evaporated to dryness at $30^{\circ} \mathrm{C}$

Esterification of fatty acids to methyl esters was carried out on the extracts evaporated to dryness using the procedure of Ferrari et al. (11) with certain modifications. Five milliliters of $0.5 \mathrm{M} \mathrm{NaOH}$ in methanol was added, and the resulting mixture was boiled for $30 \mathrm{~min}$. Subsequently, $\mathrm{BF}_{3}$ in methanol $(5 \mathrm{~mL})$ was added and the mixture was then boiled for $20 \mathrm{~min}$. After cooling, the FAMEs were extracted with $10 \mathrm{~mL}$ of hexane. The volume was reduced to $0.5 \mathrm{~mL}$ under a stream of nitrogen.

Instruments and Procedure. The experiment was performed with a Unicam 610 gas chromatograph equipped with a flame ionization detector (FID). A Hewlett-Packard 5970A mass selective detector interfaced with a Hewlett-Packard 5790 gas chromatograph was used to identify the compounds. FAMEs were separated and determined by employing an acidified polyethylene glycol capillary column MFE-1000 (30 $\mathrm{m} \times 0.25 \mathrm{~mm}, 0.25 \mu \mathrm{m}$ film thickness) from Análisis Vínicos, inserted into a split/splitless injector. A Chromate PC data system from Philips was used.

Separation of all compounds was achieved by injecting $1 \mu \mathrm{L}$ of the final extract. Analytical conditions were as follows: oven temperature, initial isotherm of $80{ }^{\circ} \mathrm{C}$ (1 min), raised to 230 ${ }^{\circ} \mathrm{C}$ at a rate of $5{ }^{\circ} \mathrm{C} / \mathrm{min}$, final isotherm at $230{ }^{\circ} \mathrm{C}(10 \mathrm{~min})$; carrier gas, nitrogen at a rate of $1.8 \mathrm{~mL} / \mathrm{min}$.; split ratio, $50: 1$; injector temperature, $290{ }^{\circ} \mathrm{C}$; detector temperature, $300{ }^{\circ} \mathrm{C}$.

Data Processing. The data were processed using the PARVUS statistical package (12). A training set (45 observations) was constructed with the ciders belonging to the 1996 and 1997 harvests, and an evaluation set (14 observations) was constructed with the ciders belonging to the 1998 harvest. From the training set we computed the decision rules for classification purposes, and the validation of these mathematical rules was realized by means of external (using the evaluation set) and internal (cross-validation) procedures. A matrix was constructed in which rows (59) represented ciders and columns (12) corresponded to fatty acids (caproic, caprilic, capric, lauric, myristic, pentadecanoic, palmitic, palmitoleic, stearic, oleic, linoleic, and linolenic acid). Samples were categorized according to the harvesting year of the apples used for cider-making as 1 (25 observations; apples harvested in 1996) and 2 (20 observations; apples harvested in 1997).

\section{RESULTS AND DISCUSSION}

Fatty Acids Analysis. Figure 1 shows a typical gas chromatogram of the FAMEs of a cider. F atty acids were identified by comparing retention times and mass spectra with those of standard methyl esters. The characteristic ions were $\mathrm{m} / \mathrm{z} 74\left(\mathrm{C}_{3} \mathrm{H}_{6} \mathrm{O}_{2}{ }^{+}\right)$and $\mathrm{m} / \mathrm{z} 87$ $\left(\mathrm{C}_{4} \mathrm{H}_{7} \mathrm{O}_{2}{ }^{+}\right)$.

Quantitative information was obtained using the internal standard method. An individual internal standard curve was generated for each fatty acid due to differences in response factors. Two internal standards were used: pelargonic (C9) and margaric acid (C17). Pelargonic acid was the most suitable one for quantification of volatile and medium-chain fatty acids (C6, C8, C10, C12, and C14) and margaric acid for the longer fatty acids (C15, C16, C16:1, C18, C18:1, C18:2, and C18:3). A good correlation of the standards and corresponding peak areas ( $r=0.999)$ over the range between the detection limits and samples content was established for all fatty acids. The validity of the regression model used was checked with the lack-of-fit and the Fisher tests (13).

The detection limit was cal culated by injecting serial dilutions of a concentrated standard mixture followed by the preparation of calibration plots (peak height

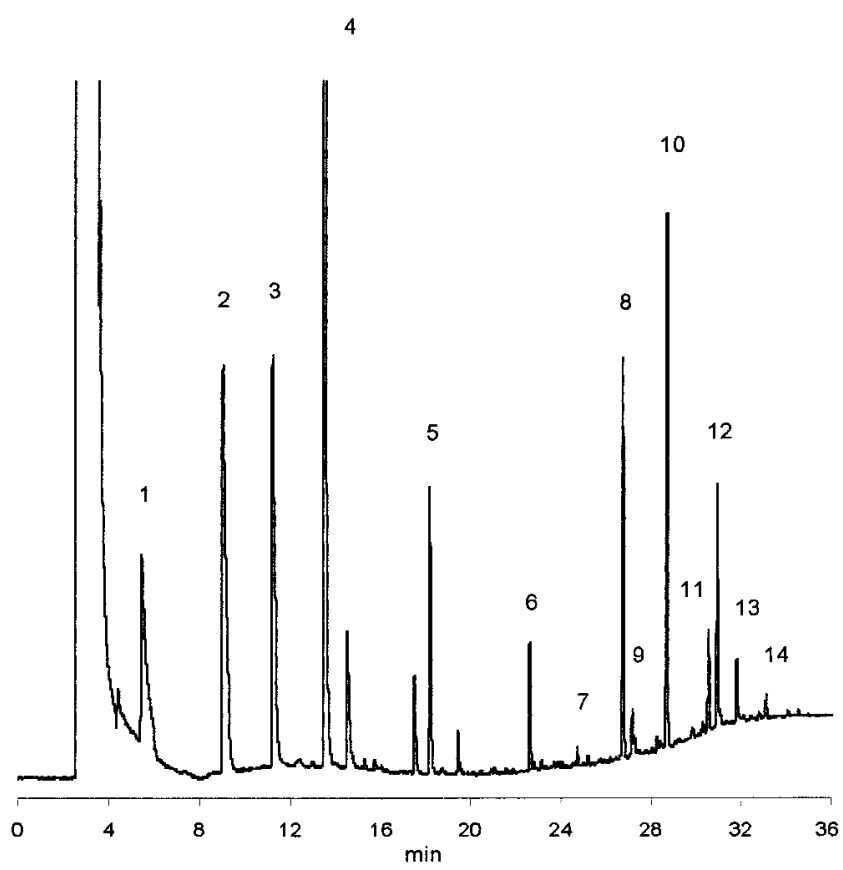

Figure 1. Chromatogram of fatty acid methyl esters of a cider: 1, caproi c; 2 , caprylic; 3, pelargonic (ISTD); 4, capric; 5 , lauric; 6, myristic; 7, pentadecanoic; 8, margaric (ISTD); 9, palmitic; 10, palmitoleic; 11, stearic; 12 , oleic; 13, Iinoleic; 14 , linolenic.

Table 1. Recovery, Reproducibility, and Detection Limits of Fatty Acids

\begin{tabular}{lrcc}
\hline \multicolumn{1}{c}{ fatty acid } & $\begin{array}{c}\text { \% recovery } \pm \\
\text { RSD }\end{array}$ & $\begin{array}{c}\text { reproducibility } \\
\text { RSD (\%) }\end{array}$ & $\begin{array}{c}\text { detection } \\
\text { limit (ng) }\end{array}$ \\
\hline caproic acid & $97 \pm 5$ & 1.2 & 1.2 \\
caprylic acid & $101 \pm 4$ & 1.3 & 1.5 \\
capric acid & $99 \pm 5$ & 5.9 & 1.8 \\
lauric acid & $100 \pm 4$ & 5.6 & 1.3 \\
myristic acid & $95 \pm 4$ & 0.8 & 0.8 \\
pentadecanoic acid & $92 \pm 5$ & 4.0 & 0.7 \\
palmitic acid & $99 \pm 4$ & 4.2 & 1.4 \\
palmitoleic acid & $102 \pm 3$ & 5.6 & 1.9 \\
stearic acid & $105 \pm 3$ & 4.9 & 3.6 \\
oleic acid & $96 \pm 3$ & 1.4 & 1.7 \\
linoleic acid & $92 \pm 4$ & 4.4 & 1.7 \\
linolenic acid & $102 \pm 5$ & 3.1 & 2.5
\end{tabular}

versus concentration injected), which were extrapolated to a signal-to-noise ratio of 3 standard deviations (SD) so as to assign the limits of detection. Table 1 shows the detection limits for each fatty acid.

Method reproducibility was cal culated for five analyses of the same sample and expressed as relative standard deviations (RSD). The reproducibility was $<6 \%$ for all fatty acids.

Recovery studies were carried out to determine the accuracy of the method. Known amounts (between 50 and $150 \%$ of the amount found in the cider) of each fatty acid were added to a sample, and the resulting spiked samples were subjected to the entire analytical sequence. All analyses were carried out in triplicate at three concentration levels. The average recoveries ranged between 92 and 105\%, testifying to the accuracy of the proposed method. Results are shown in Table 1.

Table 2 reports the mean content of 25 analytical determinations from the 1996 harvest, 20 from that of 1997, and 14 from that of 1998. Twel ve fatty acids were identified and quantified, four of these (caproic, caprylic, capric, and palmitic) constituting the major fatty acids in cider. Saturated acids predominate over unsaturated 


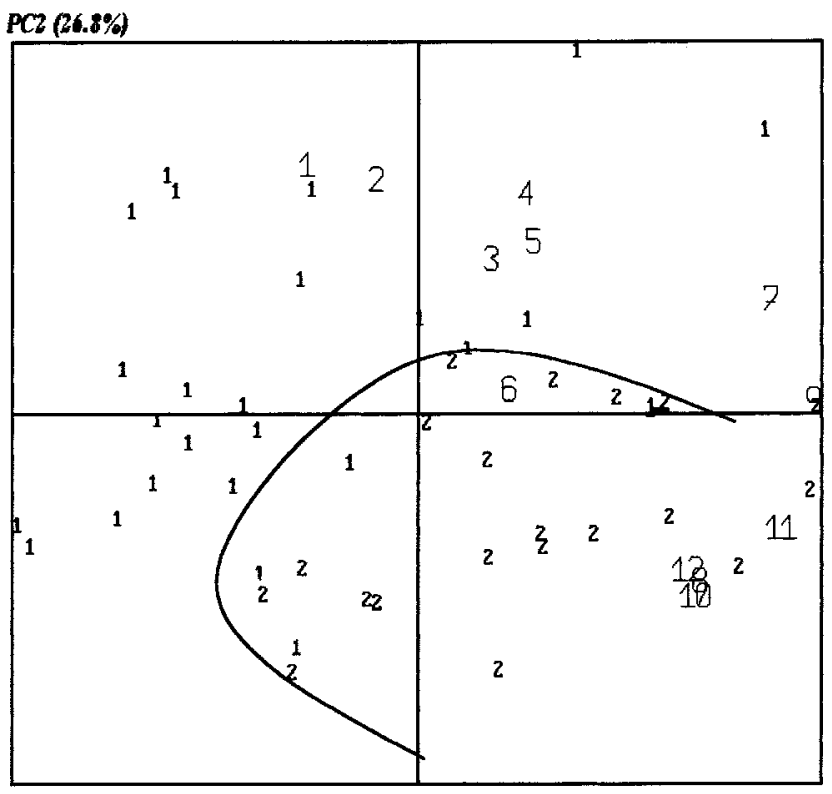

PCI (30\%)

Figure 2. Projection of the variables and samples onto the plane formed by the two first varivectors: (1) cider made from apples harvested in 1996; (2) cider made from apples harvested in 1997; 1, caproi c; 2, caprylic; 3, capric; 4, lauric; 5, myristic; 6 , pentadecanoic; 7, palmitic; 8, palmitoleic; 9 , stearic; 10, oleic; 11, linoleic; 12, linolenic.

Table 2. Total Concentration of Fatty Acids in Cider

\begin{tabular}{|c|c|c|c|c|c|c|}
\hline \multirow[b]{2}{*}{ fatty acid } & \multicolumn{2}{|c|}{1996 harvest } & \multicolumn{2}{|c|}{1997 harvest } & \multicolumn{2}{|c|}{1998 harvest } \\
\hline & $\mathrm{mg} / \mathrm{L}$ & $\%$ & $\mathrm{mg} / \mathrm{L}$ & $\%$ & $\mathrm{mg} / \mathrm{L}$ & $\%$ \\
\hline caproic (C6) & 3.2 & 24.2 & 1.5 & 13.2 & 3.0 & 19. \\
\hline caprylic (C8) & 2.8 & 21.2 & 2.0 & 17.5 & & 13 \\
\hline capric (C10) & 3.5 & 26.5 & 3.6 & 31.6 & 5. & 34 \\
\hline lauric (C12) & 0.7 & 5.3 & 0.5 & 4.4 & 0.7 & 4 \\
\hline myristic (C14) & $0.8(-19-1)$ & 4.5 & 0.4 & 3.5 & 0.5 & \\
\hline pentadecanoic (C15) & 0.1 & 0.8 & 0.1 & 0.9 & 0.1 & \\
\hline palmitic (C16) & 1.4 & 10.6 & 1.7 & 14.9 & 2.1 & 13 \\
\hline palmitoleic $(16: 1)$ & 0.1 & 0.8 & 0.2 & 1.8 & 0.1 & 0 \\
\hline stearic (C18) & 0.3 & 2.3 & 0.5 & 4.4 & 0.5 & \\
\hline oleic (C18:1) & 0.2 & 1.5 & 0.4 & 3.5 & 0.7 & \\
\hline linoleic (C18:2) & 0.2 & 1.5 & 0.4 & 3.5 & 0.1 & 0 \\
\hline linolenic (C18:3) & 0.1 & 0.8 & 0.1 & 0.9 & 0.1 & \\
\hline total & 13.2 & & 11.4 & & 15.2 & \\
\hline
\end{tabular}

ones, the major component being capric followed by caprylic, caproic, and palmitic acid. Among unsaturated acids, the major one was linoleic acid.

Statistical Analysis. Data Univariate Analysis. Before the multivariate techniques were applied to characterize the ciders, a univariate analysis was carried out. We computed the univariate weights (Fisher weights, FW) of the original variables (fatty acids) from the ratio between intercentroid variance and intracategory variances. The variable that provided the best separation between the two classes was palmitoleic acid ( $F W=1.49$ ). However, the sole use of this variable for classification purposes did not allow us to differentiate both categories.

Multivariate Analysis. Principal component analysis (PCA) was used to ascertain the structure of the data and to reduce the number of variables. The number of significant components was evaluated on the basis of double-cross-validation of the components by means of the NIPALS method (14), using five cancellation groups. Two predictive components that accounted for $56.8 \%$ of the variance were chosen. At the same time, a raw
Table 3. Prediction Matrix for LDA Technique Validation (Three Groups for Cancellation)

\begin{tabular}{lcccc}
\hline & \multicolumn{2}{c}{ assigned category } & \\
\cline { 2 - 3 } true category & 1996 harvest & 1997 harvest & hits (\%) \\
\hline 1996 harvest & 23 & 2 & 92 \\
1997 harvest & 2 & 18 & 90 \\
overall & & & 91
\end{tabular}

varimax rotation (orthogonal rotation) technique was used to maximize the simplicities of the two predictive factors and to search the latent structure of the data. As may be seen in Figure 2, a structurization of the data can be visualized because the ciders made from apples harvested in 1997 (referred to as 2) were placed in the bottom right-hand corner of the factorial plane formed by the first two predictive varivectors, these observations being closely related to fatty acids of a higher molecular mass (palmitic, palmitoleic, stearic, oleic, linoleic, and linolenic acid).

Once the structure of the data had been visualized, we used linear discriminant analysis (LDA) to classify the ciders according to the categorization criterion described under Data Processing. LDA computes a mathematical decision rule that allows the category to which a sample belongs to be ascertained. When all of the objects were included in the training set, $100 \%$ of classification hits were obtained. The LDA method was validated by means of a cross-validation procedure using three groups for cancellation and an external procedure using the evaluation set (1998 harvest). Table 3 presents the prediction matrix for each class; as we can see, the prediction hits were $91 \%$, and the percentage of prediction hits for the evaluation set using the mathematical rule computed from data of 1996 and 1997 harvest years (training set) was $86 \%$. Taking into account the prediction hits (91\%) obtained by means of the internal validation (cross-validation procedure), the prediction hits (86\%) for external validation, and the classification hits $(100 \%)$ for the training set, we can conclude that the LDA procedure computed is sufficiently robust for classification purposes of ciders on the basis of fatty acid profile and harvest year.

At the same time, to select the most rel evant variables for classification purposes, a stepwise LDA technique was used. To do this, we used the minimization of Wilks' lambda criterion and an $\mathrm{F}$ test at a confidence level of $>90 \%$ (an F-to-enter of 3.84 and an F-to-remove of 2.71). The selected variables were palmitoleic > pentadecanoic $>$ linoleic $>$ myristic $>$ linolenic, for which a Wilk's lambda of 0.24 had been detected; thus, $76 \%$ of total variance was explained by within-group differences.

At the same time, we have also used another classification method (Bayesian analysis) to compare the results obtained with the LDA method. All ciders were correctly classified (100\% hits); model 1 (1996 harvest year) includes $92 \%$ (sensitivity $=92$ ) of the observations assigned to this model and $15 \%$ (specificity $=85$ ) of ciders bel onging to model 2 (1997 harvest year); however, model 2 includes all ciders assigned to this model (sensitivity $=100$ ) and rejects all ciders belonging to model 1 (specificity $=100$ ). The internal validation of the Bayesian analysis, using five groups for cancellation, allowed us correctly to predict $82 \%$ of samples.

Conclusions. It can be affirmed that GC-FID may be successfully used for the qualitative and quantitative determi nation of fatty acids in cider. The derivatization reaction used produced total methylation of the free and 
bound fatty acids in $50 \mathrm{~min}$. Method validation (detection limits, reproducibility, and recovery) showed that the analytical procedure permits the quantification of fatty acids in cider.

The use of a fatty acid profile together with chemometric techniques such as PCA, LDA, and Bayesian analysis allowed us to differentiate ciders made from apples harvested in the Asturias region from those made from apples harvested outside this region.

\section{LITERATURE CITED}

(1) Shinohara, T. Gas-Chromatographic Analysis of Volatile Fatty Acids in Wine. Agric. Biol. Chem. 1985, 49, 175.

(2) Ratledge, C.; Dickinson, F. M. Lipids and Fatty Acids as Potential Flavour Components using Microbial Systems. Bioflavour '95 1995, 153-166.

(3) Lafon-Lafourcade, S.; Geneix, C.; Ribéreau-Gayon, P. Inhibition of Alcoholic Fermentation of Grape Must by Fatty Acids Produced by Yeast and Their Elimination by Yeast Ghosts. Appl. Environ. Microbiol. 1984, 47, 1246-1249.

(4) Geneix, C.; Lafon-Lafourcade, S.; Ribéreau-Gayon, P. Effect des Acides Gras sur la Viabilité des Populations de Saccharomyces cerevisiae. C. R. Acad. Sci. 1983, 296, 943-947.

(5) Dussaud, A.; Robillard, B.; Carles, B.; Duteurtre, B.; Vignes-Adler, M. Exogenous Lipids and Ethanol Influences on the Foam Behavior of Sparkling Base Wines. J. Food Sci. 1994, 59, 148-167.

(6) Maujean, A.; Poinsaut, P.; Dantan, H.; Brissonet, F.; Cossiez, E. Study of the Performance and Quality of the F oam in Sparkling Wines. II. Perfecting of the Measuring Technique for Foaming Abality, Performance and Stability of the Foam in Sparkling Wines. Bull. O.I.V. 1990, 711-712, 405-427.

(7) Brissonet, F.; Maujean, A. I dentification of Some F oam-
Active Compounds in Champagne Base Wines. Am. J . Enol. Vitic. 1991, 42, 97-102.

(8) Pueyo, E.; Martín-Alvarez, P.J .; Polo, M. C. Relationship between foam characteristics and chemical composition in wines and cavas (sparkling wines). Am. J . Enol. Vitic. 1995, 46, 518-524.

(9) García-López, C.; Grané-Teruel, N.; Berenguer-Navarro, V.; García-García, J .; Martín-Carratalá, M. L. Major Fatty Acid Composition of 19 Almond Cultivars of Different Origins. A Chemometric Approach. J . Agric. Food Chem. 1996, 44, 1751-1755.

(10) Martín-Carratalá, M. L.; García-López, C.; BerenguerNavarro, V.; Grané-Teruel, N. New Contribution to the Chemometric Characterization of Almond Cultivars on the Basis of Their Fatty Acid Profiles. J . Agric. Food Chem. 1998, 46, 963-967.

(11) Ferrari, G.; Meunier, J. M.; Feuillat, M. Determination of Wine and Yeast Fatty Acids. Sci. Aliments 1987, 7, 61-76.

(12) Forina, M.; Leardi, R.; Lanteri, S.; Armanino, C. In PARVUS. An ExtendablePackage of Programs for Data Exploration, Classification and Correlation; Elsevier: Amsterdam, The Netherlands, 1998.

(13) Draper, N. R.; Smith, H. Fitting a straight line by least squares. In Applied Regression Analysis; Wiley: New York, 1981; pp 22-40.

(14) Meloun, M.; Militký, J .; Forina, M. Exploratory and factor analysis of multivariate data. In Chemometrics for Analytical Chemistry. Vol. 1: PC-Aided Statistical Data Analysis; Masson, M., Ed.; Ellis Horwood: New York, 1992; pp 279-282.

Received for review August 9, 2000. Revised manuscript received December 11, 2000. Accepted December 17, 2000. This work was made possible by financial support from the CICYT (ALI 96-1219-C02-02).

J F 001006A 